\title{
La Bretagne à travers les revues et la littérature scoutes (vers 1930-vers 1960)
}

\section{Christophe Carichon}

\section{(2) OpenEdition}

Journals

\section{Édition électronique}

URL : http://journals.openedition.org/abpo/1288

DOI : $10.4000 /$ abpo. 1288

ISBN : 978-2-7535-1494-2

ISSN : 2108-6443

\section{Éditeur}

Presses universitaires de Rennes

Édition imprimée

Date de publication : 20 juillet 2004

Pagination : 145-166

ISBN : 978-2-7535-0031-0

ISSN : 0399-0826

\section{Référence électronique}

Christophe Carichon, « La Bretagne à travers les revues et la littérature scoutes (vers 1930-vers 1960) ", Annales de Bretagne et des Pays de l'Ouest [En ligne], 111-2 | 2004, mis en ligne le 20 juillet 2006, consulté le 20 avril 2019. URL : http://journals.openedition.org/abpo/1288 ; DOI : 10.4000/ abpo. 1288 


\title{
La Bretagne à travers les revues et la littérature scoutes (vers 1930-vers 1960)
}

\author{
Christophe CARICHON \\ Docteur en histoire, chercheur associé au CRBC \\ enseignant à Angers
}

\begin{abstract}
"La Bretagne est très attachée à ses traditions. Le Breton est peu porté vers l'instruction, le confort et l'industrie. Doué d'une opiniâtreté peu commune, d'une endurance extrême, c'est un marin et un pêcheur hors ligne. Le Breton est très catholique avec une tendance aux superstitions populaires ${ }^{1}$."
\end{abstract}

La Bretagne est une terre d'élection du scoutisme ${ }^{2}$, cette méthode d'éducation basée sur le jeu et le service mise au point par un général de l'empire britannique, Robert Baden-Powell, au début du xx' siècle. C'est en 1909 que la première unité scoute, d'inspiration protestante, est fondée en Bretagne, à Nantes, au sein de la Mission populaire évangélique. Quelques années plus tard, des troupes d'Éclaireurs de France, scoutisme non confessionnel, naissent dans diverses localités bretonnes à Lorient et Saint-Servan (1912), Saint-Brieuc et Rennes (1913), et Morlaix (1914). Enfin, il faut attendre l'après-guerre pour qu'un scoutisme catholique trouve sa place auprès des mouvements déjà existants. Dans les années vingt, des unités de Scouts de France (garçons) et de Guides de France (filles) sont créées dans les cinq diocèses de Bretagne. Très rapidement, ces derniers arrivés supplantent numériquement les précédents.

Terre de scoutisme de par ces nombreuses et anciennes fondations, la Bretagne est aussi une région de prédilection pour les camps et les voyages d'exploration des troupes étrangères à la région. Dès les origines, avec un surcroît d'intérêt à partir du milieu des années trente, le scoutisme (surtout catholique) incite à la connaissance des " petites patries. " Les articles des revues des mouvements se font largement l'écho de cette politique des terroirs, participant alors à la construction de représentations mentales

1. "La Bretagne ", L'Escoute, n 183 , mars 1943, p. 12. L'Escoute est le titre de la revue des Scouts de France durant une partie de la guerre.

2. Cette étude est tirée de notre thèse d'histoire, Le scoutisme catholique en Bretagne des origines aux années 1970, soutenue à l'Université de Bretagne occidentale (Brest) en 2002. 
particulières auprès des lecteurs. Plus importante encore apparaît dans cette optique l'étude des "romans scouts ", genre littéraire né vraiment à la fin des années vingt et dont le succès grandissant auprès des jeunes, filles et garçons, scouts ou non, a pu jouer un rôle dans la formation de l'imaginaire et l'exaltation d'un idéal. Cela dit, ce qui nous intéresse ici n'est pas tant la littérature scoute et les valeurs qu'elle diffuse, comme cela a déjà été étudié largement ${ }^{3}$, mais bien la Bretagne et les Bretons au travers de cette littérature. En suivant quelques patrouilles dans leurs pérégrinations, une Bretagne se révèle, contrée fascinante et mystérieuse, parfois aux frontières du réel, compagne idéale du grand jeu à la scoute, espace des plus typiques pour qui sait y trouver l'aventure.

\section{Bretagne et Bretons dans les publications nationales}

L'un des premiers articles relatifs à la Bretagne, si ce n'est le premier, paraît dans Le Scout de France à l'automne 1926 sous la plume de Marcel Forestier. Le futur aumônier général des Scouts de France n'est encore que le chef laïc de la troupe Saint-Rémi $1^{\text {re }}$ Villemomble, lorsqu'il donne dans la revue le compte rendu du camp itinérant de sa troupe dans le pays de Vannes ${ }^{4}$. Accueillis par le clergé local (collège Saint-François-Xavier, Grand séminaire, religieuses), les Parisiens apprécient volontiers « la bonhomie et l'inaltérable hospitalité bretonne " tout au long d'un périple qui les conduits de Vannes, cité de saint Vincent Ferrier " un vrai routier ${ }^{5}$ celui-là et digne de sa badge de secourisme puisque thaumaturge ", à l'île aux Moines, de Port-Navalo à Carnac, de l'abbaye de Kergonan à la basilique de Sainte-Anne-d'Auray " métropole de la Foi bretonne. " Au travers de ce premier article transparaissent de nombreux aspects de la Bretagne et des Bretons tels qu'ils sont et tels que peuvent les désirer des Scouts de France avides d'authenticité catholique et française. Deux ans plus tard, les cadets du Père Doncœur ${ }^{6}$ en leur " route de Bretagne " n'en demanderont pas

3. GAUTHÉ, Jean-Jacques, "Les travaux universitaires consacrés au scoutisme ", Mille neuf cent sept, bulletin du Réseau de recherches interdisciplinaires sur le scouting et les mouvements de jeunesse, $n^{\circ} 4$, mars 2002, relève sept maîtrises et deux thèses sur le sujet dont le travail de Christian GUÉRIN, Éclaireurs Scouts de France et Signe de Piste, histoire d'un système de représentations (1920-1964), thèse d'état en histoire, Paris X, 1993, qui consacre le tome trois à la littérature scoute. Il faut signaler aussi l'organisation par le Comité pour la promotion du scoutisme en Europe (COPSE) du colloque Le Roman scout (1927-1962) : un genre littéraire?, Actes du colloque de Strasbourg publiés par Ianus Bifrons, Revue universitaire de l'adolescence, $n^{\circ}$ 4/1992, Nancy, Presses universitaires de Nancy, 1993.

4. Girafe Prudente (Marcel Forestier), "Camp du Morbihan ", Le Scout de France, n ${ }^{\circ} 58$, octobre 1926, p. 14-15. Le RP Marcel-Denys Forestier (1896-1976) sera aumônier général des Scouts de France de 1936 à 1955.

5 . Un routier est un scout aîné.

6. Paul Doncœur (1880-1961), aumônier militaire, fondateur du mouvement de jeunesse des "Cadets", aumônier national de la Route des Scouts de France, célèbre pour sa lettre ouverte à Édouard Herriot " Nous ne partirons pas " (octobre 1924) lorsque le cartel des gauches voulait renvoyer à l'étranger les membres des congrégations religieuses. 
La Bretagne à travers les revues et la littérature scoutes

\begin{tabular}{|c|c|c|}
\hline Auteur & Titre de l'article & Référence \\
\hline \multicolumn{3}{|l|}{$1926-1930$} \\
\hline $\begin{array}{l}\text { Girafe Prudente } \\
\text { (Marcel Forestier) }\end{array}$ & "Camp du Morbihan" & $\begin{array}{l}\text { Le Scout de France, } \mathrm{n}^{\circ} 58, \\
\text { octobre } 1926\end{array}$ \\
\hline Jos Le Doaré & « La Légende des Rochers » & $\begin{array}{l}\text { Le Scout de France, } \mathrm{n}^{\circ} 120 \text {, } \\
1^{\mathrm{er}} \text { novembre } 1930\end{array}$ \\
\hline Jos Le Doaré & "Le Pont de Plougastel » & $\begin{array}{l}\text { Le Scout de France, } \mathrm{n}^{\circ} 120 \\
1^{\mathrm{er}} \text { novembre } 1930\end{array}$ \\
\hline \multicolumn{3}{|l|}{ 1931-1935 } \\
\hline $\begin{array}{l}\text { Abeille Joyeuse } \\
\text { des Monts d'Arré }\end{array}$ & " Le Géant de Saint-Herbot " & $\begin{array}{l}\text { Le Scout de France, n }{ }^{\circ} 152, \\
1^{\mathrm{er}} \text { avril } 1932\end{array}$ \\
\hline $\begin{array}{l}\text { Abeille Joyeuse } \\
\text { des Monts d'Arré }\end{array}$ & $\begin{array}{l}\text { "La Destruction } \\
\text { de la ville d'Ys " (1) }\end{array}$ & $\begin{array}{l}\text { Le Scout de France, } \mathrm{n}^{\circ} 155 \text {, } \\
15 \text { mai } 1932\end{array}$ \\
\hline $\begin{array}{l}\text { Abeille Joyeuse } \\
\text { des Monts d'Arré }\end{array}$ & $\begin{array}{l}\text { "La destruction } \\
\text { de la ville d'Ys " (2) }\end{array}$ & $\begin{array}{l}\text { Le Scout de France, } n^{\circ} 156, \\
1^{\text {er }} \text { juin } 1932\end{array}$ \\
\hline XXX & "Chant du soir breton" & Le Scout de France, $1^{\mathrm{er}}$ août 1932 \\
\hline Pierre Reilliem & $\begin{array}{l}\text { "La Légende du monument } \\
\text { de Gueriec " }\end{array}$ & $\begin{array}{l}\text { Le Scout de France, n }{ }^{\circ} 170, \\
1^{\text {er }} \text { février } 1933\end{array}$ \\
\hline $\mathrm{XXX}$ & $\begin{array}{l}\text { "Le Chevalier d'émeraude " (1), } \\
\text { légende de la Table ronde }\end{array}$ & Scout, $\mathrm{n}^{\circ} 18,5$ octobre 1934 \\
\hline $\mathrm{XXX}$ & $\begin{array}{l}\text { "Le Chevalier d'émeraude " (2), } \\
\text { légende de la Table ronde }\end{array}$ & Scout, $\mathrm{n}^{\circ} 20,5$ novembre 1934 \\
\hline Charles Corre & "La Messe des âmes" & Scout, $\mathrm{n}^{\circ} 45,20$ novembre 1935 \\
\hline Abbé Maréchal & "Kousk Breiz Izel » & Scout, $\mathrm{n}^{\circ} 45,20$ novembre 1935 \\
\hline Jos Le Doaré & "La Gavotte de Quimper» & Scout, $\mathrm{n}^{\circ} 45,20$ novembre 1935 \\
\hline Anatole Le Braz & "Le Tombeau de Gwenc'hlan» & Scout, $\mathrm{n}^{\circ} 45,20$ novembre 1935 \\
\hline \multicolumn{3}{|l|}{$1936-1940$} \\
\hline Georges Cerbelaud-Salagnac & $\begin{array}{l}\text { "Avec le Coëtquen, } \\
\text { frégate de dix huit canons " }\end{array}$ & Scout, $\mathrm{n}^{\circ} 59,20$ juin 1936 \\
\hline XXX & $\begin{array}{l}\text { "Alain Guyader " } \\
\text { juin-juillet } 1936\end{array}$ & La Guide de France, \\
\hline Jos Le Doaré & $\begin{array}{l}\text { "Le Pardon de la nuit } \\
\text { en Cornouaille" }\end{array}$ & Scout, $\mathrm{n}^{\circ} 63,20$ août 1936 \\
\hline Jos Le Doaré & "Par Monts et plaines" & Scout, ${ }^{\circ} 68,5$ novembre 1936 \\
\hline $\mathrm{XXX}$ & "Salut à Notre-Dame d'Armor " & Scout, $\mathrm{n}^{\circ} 70,5$ décembre 1936 \\
\hline $\mathrm{XXX}$ & "Le Tournoi de Rennes" & Scout, $\mathrm{n}^{\circ} 79,20$ avril 1937 \\
\hline $\mathrm{XXX}$ & "La Légende de Port-Blanc" & Scout, $\mathrm{n}^{\circ} 81,20$ mai 1937 \\
\hline $\mathrm{XXX}$ & "L'Aventure du petit Gab" & Scout, $\mathrm{n}^{\circ} 92,5$ novembre 1937 \\
\hline \multicolumn{3}{|l|}{ 1941-1944 } \\
\hline Pierre Joubert & Couverture de la revue & Scout, $\mathrm{n}^{\circ}$ 172, mars 1942 \\
\hline $\mathrm{XXX}$ & "Le tournoi de Rennes" & Scout, $\mathrm{n}^{\circ} 173$, avril 1942 \\
\hline $\mathrm{XXX}$ & "La Bretagne" & L'Escoute, mars 1943 \\
\hline \multicolumn{3}{|l|}{$1945-1960$} \\
\hline $\mathrm{XXX}$ & « Notre-Dame du Folgoat » & Guide de France, n ${ }^{\circ}$ 199, mai 1946 \\
\hline $\mathrm{XXX}$ & « Le connétable de Clisson" & Louveteau, $\mathrm{n}^{\circ} 7,1^{\mathrm{er}}$ avril 1948 \\
\hline $\begin{array}{l}\text { Diverses compagnies } \\
\text { de Bretagne }\end{array}$ & "Les sanctuaires mariaux" & $\begin{array}{l}\text { Guide de France, } \mathrm{n}^{\circ} 1, \\
\text { octobre } 1954\end{array}$ \\
\hline $\begin{array}{l}\text { Chantal-Annick, équipe } \\
\text { des Hermines de la } 9^{\mathrm{e}} \text { Rennes }\end{array}$ & "Rennes, ma ville" & Guide de France, ${ }^{\circ} 7$, avril 1958 \\
\hline
\end{tabular}


davantage $^{7}$. De la Bretagne, du golfe du Morbihan en fait, les Parisiens remportent l'image d'une contrée exotique (mer, île, autochtones) marquée par l'histoire (préhistoire, Révolution) et l’Église. Voici qui consacre vite la Bretagne comme une province de prédilection pour les camps scouts, à l'instar du Périgord, de l'Alsace ou des Pyrénées. Par la suite, plusieurs textes confirment cette image typique vue de France mais donnent aussi une peinture plus authentique si l'on en juge par les revues dépouillées dans le tableau ci-dessus.

Au regard de cette liste, un premier constat s'impose : l'extraordinaire disproportion entre l'entre-deux-guerres, la guerre et l'après-guerre. Tous les articles régionalistes sur la Bretagne paraissent dans la revue Le Scout de France puis Scout avant 1944. En proportion, nous n'avons trouvé qu'un article dans la presse guide. Après la guerre, le thème s'épuise puis disparaît totalement à partir du début des années cinquante, malgré l'offensive du journal Guide de France jusqu'au milieu de la décennie. Les provinces sont alors uniquement mentionnées sous la rubrique "Vie du Mouvement " et félicitées pour les créations d'unités nouvelles. Les raisons de ce tarissement sont très simples : le Quartier général a changé de mode considérant que l'aspect régionaliste et le rappel à l'histoire médiévale, très en vogue auparavant, ne convenaient plus aux garçons. Les montres des chevaliers se sont effacées devant les sticks des commandos parachutistes (années cinquante) puis devant les premières caravanes des pionniers (années soixante) à vocation beaucoup plus internationale que la défense de la « doulce France " et de Breiz ma Bro du temps jadis ${ }^{8}$.

Revenons alors dans les années trente : le commissaire de province de Bretagne ${ }^{9}$ Charles Picquenard (dit " Abeille Joyeuse des Monts d'Aré ") et un commissaire du district de Cornouaille, le photographe châteaulinois Jos Le Doaré, monopolisent largement la plume des scouts de Bretagne dans les revues. Personnage original s'il en est ${ }^{10}$, le docteur Picquenard, " membre du collège des Bardes de petite Bretagne ", se contente cependant de folklore classique avec ces deux contes que sont Le géant de SaintHerbot et La destruction de la ville d'Ys. Jos Le Doaré (1904-1976), chef scout mais aussi illustrateur et photographe de renom, entraîne quant à

7. Voir l'article de Dominique Avon, "Quête de chrétienté, été 1928 : la "route Doncoeur" en Bretagne ", dans TranvouEz, Yvon (dir.), Scoutisme en Bretagne, Scoutisme breton? (vers 1930-vers 1960), Kreiz 7, Brest, CRBC-UBO, 1997, p. 27-51.

8. Sur ce sujet voir Gilles SAINT-AUBIN, "Le langage du signe, du scout-chevalier au pionnier, évolution des modèles éducatifs des Scouts de France ", dans CHOLVY, Gérard et CHEROUTRE, Marie-Thérèse (dir.), Le scoutisme, quel type d'hommes?, Quel type de femmes?, Quel type de chrétiens?, Paris, Cerf, 1994.

9. Un commissaire scout est un adulte dirigeant une subdivision géographique qui peut aller de la ville à plusieurs départements ou diocèses.

10. Docteur en médecine, ancien combattant volontaire de 1914-1918, érudit botaniste et zoologiste, membre de nombreuses sociétés scientifiques, vice-président de la Société archéologique du Finistère, poète, conteur, membre fondateur du " Gorsedd des bardes ", vice-président de l'Union régionaliste bretonne, Charles Picquenard (1872-1940) a 52 ans, et une vie déjà bien remplie, quand il se lance dans le scoutisme. 
lui le lecteur dans la Bretagne de son temps. Ses articles sur la Cornouaille sont des reportages plus que des histoires. Toutefois, Il n'en propose pas moins à la revue les contes, légendes et chants d'Anatole Le Braz ${ }^{11}$, de Charles Corre ${ }^{12}$ et de l'abbé Maréchal, et compose largement la partie "Bretagne " du numéro de novembre $1935^{13}$. Jusqu'aux années cinquante, les photos de sites et de monuments bretons des "Éditions Jos " feront encore quelques belles pages des publications scoutes et guides. Deux derniers auteurs sont des chefs scouts plus connus : Le dessinateur Pierre Joubert qui illustre avantageusement les contes celtes et bretons et l'auteur pour la jeunesse Georges Cerbelaud-Salagnac qui dans cette aventure maritime de Duguay-Trouin, vaillant commandant de 19 ans, laisse libre court à son amour de la Bretagne et de la mer en contant le combat entre le corsaire de Louis XIV et cinq bâtiments de la perfide Albion ${ }^{14}$. Il faut signaler enfin que les Éclaireurs de France (scoutisme masculin non confessionnel) consacrent eux aussi un numéro entier de leur revue à la Bretagne $^{15}$.

Toutefois, malgré ces louables efforts de Jos Le Doaré, du docteur Picquenard et de quelques autres, la Bretagne vue de France demeure surtout celle des clichés : un " pays de foi, pays des grands Pardons, des coiffes blanches et des vestes à velours noir ${ }^{16}$. "Visions qui, sans être forcément inauthentiques, n'en restent pas moins réductrices. La littérature scoute, celle des romans, donne une ampleur encore supérieure à cette mythologie semi-imaginaire.

\section{La Bretagne dans les romans scouts (1927-1964)}

"Le roman scout est un roman dans lequel le ou les principaux personnages sont scouts, qui se déroule dans un cadre scout ou dans lequel le

11. Anatole Le Braz (1859-1926), professeur de lettres à Quimper et à l'université de Rennes est connu pour avoir enquêté et collecté le patrimoine populaire oral de Basse Bretagne.

12. Conteur populaire de la fin du XIX ${ }^{\mathrm{e}}$ siècle. Il est cité par Anatole Le Braz dans $L a$ légende de la mort chez les Bretons armoricains, texte repris dans Magie de la Bretagne, t. 1, Collection Bouquins, Paris, Robert Laffont, 1994, p. 313 et p. 359.

13. Ce numéro est sous titré "Nos provinces "; un numéro du même esprit paraît deux ans plus tard ( ${ }^{\circ}$ 85, 20 juillet 1937) mais il ne contient aucun article sur la Bretagne.

14. D'après Jacqueline et Bernard LE NAIL, Dictionnaire des auteurs de jeunesse de Bretagne, Collection Patrimoine littéraire de Bretagne, Spézet, Keltia Graphic, Éditions des Montagnes Noires, 2001, p. 45-46, Georges Cerbelaud-Salagnac (1906-1999) " manifesta de bonne heure une grande sympathie pour la Bretagne, adhéra à un groupe d'étudiants bretons et s'initia à la langue bretonne et, ayant choisi de faire son service national dans la marine, il opta pour le faire à Brest ". Georges Cerbelaud-Salagnac a écrit le premier roman de la collection Signe de Piste, Sous le signe de la tortue, Paris, Alsatia, 1937. Mais, sur sa quarantaine de livres publiés, il ne donna presque rien sur la Bretagne où il s'était installé, à Ploudalmézeau. Voir aussi La Gazette des Amis de l'Orme Rond, $\mathrm{n}^{\circ} 60$, mars 1999, p. 12-15.

15. L'Éclaireur de France, $\mathrm{n}^{\circ}$ 7, 5 avril 1933.

16. Loup-Blanc, " La belle vie du Général Guyot d'Asnières de Salins, Chef-Scout de France ", Le Chef, 20 novembre 1936, n 137, p. 6. 
scoutisme joue un rôle déterminant " écrit Jean-François Roussel Secrétaire général du Comité pour la promotion du scoutisme en Europe dans l'"Introduction générale " au colloque Le Roman scout (1927-1962) : un genre littéraire ${ }^{17}$ ? Pour Jean-Louis Foncine, auteur de célèbres romans scouts, cette littérature se définie aussi par l'esprit qui sous-tend une histoire caractérisée par deux mythes principaux : celui de « la bande, c'est à dire le mythe de la patrouille " et celui du " mythe de l'aventure libre dans la nature ${ }^{18}$ ".

1927 : Pierre Delsuc, 25 ans, commissaire de district et chef de la $5^{\mathrm{e}}$ Paris des Scouts de France ${ }^{19}$, donne à la revue du mouvement ce qui est considéré comme le premier roman authentiquement scout : La Rude Nuit de Kervizel. 1964 : Michel Renouard, 22 ans, chef de la $1^{\text {re }}$ Dinan, fait paraître l'un des derniers romans de la période classique du scoutisme : Lumière sur Kerlivit. Entre ces deux dates (37 ans), les auteurs de quinze romans scouts, ou guides, prennent pour cadre, avec un inégal bonheur, l'un ou l'autre des pays de Bretagne ${ }^{20}$. Après dépouillement des différentes collections de livres pour la jeunesse, nous avons dressé la liste ci-dessous que nous pensons assez juste, des romans scouts où la Bretagne tient une place essentielle dans le décor et l'intrigue de l'histoire ${ }^{21}$.

\begin{tabular}{|l|l|l|}
\hline Auteur & Titre & Référence \\
\hline $1^{\text {re }}$ période : L'avant-guerre 1927-1940 (3 romans) & \multicolumn{2}{|l|}{$\begin{array}{l}\text { Paris, Spes, 1928 } \\
\text { coll. " Des Fleurs et des Fruits " } \\
\text { et en feuilleton dans Le Scout } \\
\text { de France à partir du n } \\
\text { décembre } 1927 .\end{array}$} \\
\hline Delsuc (Pierre) & La Rude nuit de Kervizel & $\begin{array}{l}\text { En feuilleton dans Scout } \\
\text { à partir du n } 10 \text { (5 juin 1934). } \\
\text { En feuilleton dans Scout } \\
\text { à partir du n }{ }^{\circ} 151 \text { (20 avril 1940), } \\
\text { roman interrompu par la guerre }\end{array}$ \\
\hline $2^{e}$ période : L'après-guerre 1944-1950 (5 romans) & Une Ténébreuse affaire & Les Disparus de Kerlasdouès \\
\hline Dachs (Jean Busson dit ${ }^{22}$ ), & L'Étrange aventure de Carnoët & Paris-Saint Étienne, \\
\hline
\end{tabular}

17. Ianus Bifrons, op. cit, p. 178.

18. FoncINE, Jean-Louis, "La naissance du roman scout ", Ianus Bifrons, op. cit., p. 189-195.

19. Voir la notice très complète de Louis V. M. Fontaine, La mémoire du scoutisme, dictionnaire des hommes, des thèmes et des idées, Paris, Publications L. F., 1999, p. 137-139.

20. Dans les collections pour la jeunesse du Signe de Piste, de Jamboree où d'autres maisons semblables, quelques romans pour jeunes se passent en Bretagne mais ne sont pas, à proprement parler, des romans scouts. Malgré leur richesse d'évocation régionale, nous ne les retenons pas dans notre liste. Nous pensons ici spécialement à Marion Cahours, Les Chevaliers de l'île aux Pies, collection Signe de Piste, Paris, Alsatia, 1945 (Pays de Redon), à Jean-Louis Foncine, La Caverne aux épaves, collection Prince Éric, Paris, Alsatia, 1957 (Côte fouesnantaise) ou à Pierre Delsuc, L'Étrille, qui paraît en feuilleton dans Scout, du n 267 (décembre 1951) au n² 274 (juillet 1952) puis dans la collection "Jamboree ", Paris, Spes, 1953, (Les Glénans).

21. Nous avons aussi éliminé les romans où la Bretagne est simplement mentionnée au détour d'une ligne où d'un paragraphe.

22. Dachs, blaireau en allemand, est le totem scout de Jean Busson. 
La Bretagne à travers les revues et la littérature scoutes

\begin{tabular}{|c|c|c|}
\hline & & $\begin{array}{l}\text { Éditions Dumas, } \\
\text { coll. " Jeunes de France ", } 1945\end{array}$ \\
\hline Dachs (Jean Busson dit) & Traon-Ar-Rhun & $\begin{array}{l}\text { Paris-Saint-Étienne, } \\
\text { Éditions Dumas, } \\
\text { coll. « Jeunes de France », } 1946\end{array}$ \\
\hline Baux (Raymond) & Les Fantômes de la chapelle Pol & $\begin{array}{l}\text { Paris, Alsatia, } \\
\text { coll. "Signe de Piste ", } 1948\end{array}$ \\
\hline Fuval (Pierre) & L'Évadé de Coëtcarantec & $\begin{array}{l}\text { Paris, Alsatia, } \\
\text { coll. "Signe de Piste ", } 1946\end{array}$ \\
\hline Alain (Jean-Claude) & La Maison du bord des sables & $\begin{array}{l}\text { Paris, Alsatia, } \\
\text { coll. "Signe de piste ", } 1950\end{array}$ \\
\hline \multicolumn{3}{|c|}{$3^{e}$ période : Des raiders à la réforme $1950-1964$ (6 romans) ${ }^{23}$} \\
\hline Henrys (Paul) & Le Signe dans la pierre & $\begin{array}{l}\text { Paris, Alsatia, } \\
\text { coll. "Signe de Piste ", } 1952\end{array}$ \\
\hline Avron (Philippe) & Patrouille ardente & $\begin{array}{l}\text { Paris, Alsatia, } \\
\text { coll. "Signe de Piste ", } 1953\end{array}$ \\
\hline Delsuc (Pierre) & Toul Ann Héry & $\begin{array}{l}\text { Paris, Spes, } \\
\text { coll. " Jamboree ", } 1954\end{array}$ \\
\hline Delsuc (Pierre) & Gaël des Glénans & $\begin{array}{l}\text { Paris, Spes, } \\
\text { coll. « Jamboree », } 1956\end{array}$ \\
\hline Delsuc (Pierre) & L'̂lle de fer & $\begin{array}{l}\text { Paris, Spes, } \\
\text { coll. " Jamboree ", } 1960^{24}\end{array}$ \\
\hline Renouard (Michel) & Lumière sur Kerlivit & $\begin{array}{l}\text { Paris, Desclée De Brouwer, } \\
\text { coll. " Belle Humeur ", } 1964\end{array}$ \\
\hline \multicolumn{3}{|l|}{ Roman guide (1 roman) } \\
\hline $\begin{array}{l}\text { Royer (Jacqueline) } \\
\text { et La Selve (Simone) }\end{array}$ & Une fille pas comme les autres & $\begin{array}{l}\text { Paris-Tournay, Casterman, } 1953 \\
\text { Paraît d'abord en feuilleton } \\
\text { sous le titre "An Ano Kolet " } \\
\text { dans Guide de France, du n }{ }^{\circ} 243 \\
\text { (janvier 1951) au n }{ }^{\circ} 259 \\
\text { (juillet 1952) }^{25}\end{array}$ \\
\hline
\end{tabular}

L'on peut estimer à environ quatre cents le nombre maximum de romans scouts francophones parus depuis les origines, dont le quart aux deux grandes collections du Signe de Piste et de Jamboree ${ }^{26}$. Les romans scouts ayant pour cadre la Bretagne ne correspondent donc qu'à environ $4 \%$ du total, ce qui, sur la période reste conforme au poids du scoutisme de Bretagne à l'échelle nationale. Il n'y a donc ni sur représentation ni sous représentation.

23. Les raiders sont des scouts d'élite caractérisés par un haut niveau technique, moral et spirituel. Ils connaissent leurs heures de gloire pendant une quinzaine d'années jusqu'aux réformes pédagogiques et structurelles qui affectent les mouvements de scoutisme dans les années soixante.

24. Ces deux derniers romans sont relus et refondus 20 ans plus tard en Gaël des Glénans, Paris, Alsatia-Épi, coll. "Le Nouveau Signe de Piste ", 1978. C'est cette dernière édition que nous étudions.

25. Précédemment, à la rentrée 1945, le journal Guide de France avait entrepris la publication d'un roman guide dont l'action se déroulait en Bretagne : Le Pen-Du. Un seul chapitre, non signé, était paru. Voir Guides de France, n 194, octobre-novembre 1945, p. 6-7.

26. Voir la seconde étude de Jean-François Roussel « Bilan de recherches sur le roman scout francophone ", Ianus Bifrons, op. cit., p. 241-252. 


\section{Les auteurs}

Les auteurs de ces quinze romans sont onze, dont un duo. Sur certains d'entre eux il a été possible de glaner quelques éléments biographiques mais d'autres restent totalement inconnus.

\begin{tabular}{|c|c|c|}
\hline Nom & Liens avec la Bretagne & Scoutisme \\
\hline $\begin{array}{l}\text { Pierre Delsuc } \\
(1902-1986) \\
\text { Avocat }\end{array}$ & $\begin{array}{l}\text { Nombreux camps. En retraite, } \\
\text { se fixe à Sainte-Marine (29) } \\
\text { Décédé à Pont-L'Abbé }\end{array}$ & $\begin{array}{l}\text { Entré à la } 5^{\mathrm{e}} \text { Paris SDF (1921), } \\
\text { chef de troupe puis commissaire } \\
\text { pour la zone nord (occupation), } \\
\text { commissaire général } \\
(1944-1946), \text { se retire en } 1960\end{array}$ \\
\hline Les Gerfaux & $?$ & $?$ \\
\hline Alain Breiz & $?$ & $?$ \\
\hline $\begin{array}{l}\text { Dachs (Jean Busson dit) } \\
\text { (Né en 1920) } \\
\text { Homme de Lettres, } \\
\text { Cadre de société }\end{array}$ & $\begin{array}{l}\text { Ses grands-parents maternels } \\
\text { sont fixés à Plougasnou. } \\
\text { Il y passe toutes ses vacances } \\
\text { depuis sa plus tendre enfance } \\
\text { et y possède toujours une } \\
\text { résidence secondaire. Prépare } \\
\text { Saint-Cyr à l'école Saint-Yves } \\
\text { de Quimper (1939-1940) }\end{array}$ & $\begin{array}{l}\text { Entré à la } 1^{\text {re }} \text { Villeneuve } \\
\text { Saint-Georges SDF (1935), } \\
\text { Routier, chef de la } 1^{\text {re }} \text { Quimper } \\
\text { (1939-1940), chef en France et } \\
\text { en Algérie }\end{array}$ \\
\hline Raymond Baux & $?$ & $?$ \\
\hline $\begin{array}{l}\text { Pierre Fuval (Paul Février dit) } \\
\text { (1906-1989) Médecin }\end{array}$ & Né à Pont-L’Abbé. & $?$ \\
\hline $\begin{array}{l}\text { Jean-Claude Alain } \\
\text { (Jean Léopold dit) } \\
\text { (Né en 1916) }\end{array}$ & & $\begin{array}{l}\text { Chef à l'Association nationale } \\
\text { des Scouts Russes. L'un des } \\
\text { fondateurs des Scouts d'Europe } \\
(1958)\end{array}$ \\
\hline Paul Henrys & $?$ & $?$ \\
\hline $\begin{array}{l}\text { Philippe Avron (né en 1928) } \\
\text { Professeur dans un centre } \\
\text { d'enfants caractériels, } \\
\text { Comédien }\end{array}$ & $\begin{array}{l}\text { Né au Croisic } \\
\text { Études au collège de Saint-Malo } \\
(1942-1946)\end{array}$ & $?$ \\
\hline $\begin{array}{l}\text { Michel Renouard } \\
\text { (Né en 1942) } \\
\text { Professeur des Universités }\end{array}$ & Né à Dinan & $\begin{array}{l}\text { Entré à la } 2^{\mathrm{e}} \text { Dinan SDF (1957), } \\
\text { chef de la } 1^{\text {re }} \text { Dinan (1962), } \\
\text { Routier }\end{array}$ \\
\hline $\begin{array}{l}\text { Jacqueline Royer et } \\
\text { Simone La Selve du Fayn, } \\
\text { Psychologue (J. R.) } \\
\text { Métiers de l'édition (S. L. S) }\end{array}$ & $?$ & $\begin{array}{l}\text { Entrées en } 1929 \text { et } 1940, \\
\text { guide aux } 3^{\mathrm{e}} \text { Nancy, } 1^{\text {re }} \text { et } \\
2^{\mathrm{e}} \text { Antibes, } 127^{\mathrm{e}} \text { Paris. } \\
\text { Rédactrices à Guide de France }\end{array}$ \\
\hline
\end{tabular}

Tous les auteurs connus sont des praticiens du scoutisme en activité et, sans risque de se tromper, on peut en dire autant des inconnus. Leurs descriptions du système, des techniques et de l'esprit prouvent largement leurs connaissances scoutes. Plusieurs, bien sûr, partent d'histoires vécues et de camps auxquels ils ont participé. C'est le cas de Michel Renouard, chef de la $1^{\text {re }}$ Dinan, qui campe à Kerlivit en Argol (juillet 1961) ${ }^{27}$; celui aussi de Jean Busson dit Dachs qui conduit le lecteur à Carnoët au dernier camp libre de la $1^{\text {re }}$ Quimper (Pâques 1940) ${ }^{28}$; celui enfin de Philippe Avron s'ins-

27. Lettre de Michel Renouard, 17 juin 1996.

28. Lettre de Jean Busson, 7 mars 1994. 
pirant de ses aventures scoutes malouines durant l'occupation (1943$1944)^{29}$. Le plus prestigieux d'entre les auteurs, et celui dont les romans portent la bretonnité la plus authentique, reste Pierre Delsuc : chef scout, commissaire au plus haut niveau, avocat, écrivain aimant à affirmer "être un de ceux qui sont venus à la littérature par le Scoutisme ${ }^{30}$ ". L'œuvre littéraire de Delsuc se divise en un grand nombre d'articles dans les revues des Scouts de France, des ouvrages de progression technique de grande valeur $^{31}$, des pièces de théâtre scout et des romans. La production romanesque de Pierre Delsuc se monte à une douzaine d'histoires. Parmi cellesci, plus de la moitié prend pour cadre la Bretagne et quatre sont des romans scouts. Les autres sont des histoires de mer dont l'action se déroule invariablement au large de Bénodet, villégiature préférée de l'auteur. Après Pierre Delsuc, Jean Busson est l'auteur dont l'œuvre est la plus marquée par la Bretagne. En 1944-1945, directeur de la collection "Jeunes de France " (chez l'éditeur Dumas) et alors aux armées, Dachs n'en fait pas moins paraître un roman tous les six mois dans l'immédiat après-guerre. La liste de ses publications est aussi importante mais peut-être plus variée et surtout toujours en renouvellement grâce aux rééditions et aux nouveaux livres du très prolifique ancien chef de la $1^{\text {re }}$ Quimper. D'ailleurs, il est le seul à avoir vécu de sa plume pendant une vingtaine d'années. Les autres auteurs ont écrit des romans scouts uniques sur la Bretagne, des romans de jeunesse qu'ils n'ont pas reniés devenus adultes.

\section{Les romans}

Ces quinze romans sont inégaux. Non pas dans leur style, voire dans l'intérêt de l'intrigue qu'il ne nous appartient pas de juger, mais dans leur présentation du cadre géographique et historique dans lequel ils s'inscrivent : la Bretagne. Yvon Tranvouez, dans son introduction à la journée d'études de Kerjean, a parlé de « la place bien particulière » occupée par la Bretagne dans "l'imaginaire scout " :

"Bretagne, mythe du scoutisme? [...] tout suggère de répondre oui. Parce qu'ils sont en masse ailleurs, les scouts viennent nombreux ici à l'occasion de ces moments privilégiés que sont le camp ou la routé de l'été. Mais pour y trouver quoi? D'abord la nature, et quelle nature! La variété de ses paysages, l'instabilité et la vigueur de son climat, la rudesse de ses habitants et l'hermétisme de leur langage, font de la Bretagne une contrée fascinante, à la fois attirante et étrange, avec cette part du mystère qui sied au jeu scout $^{32}$."

29. Lettre de Philippe Avron, 16 avril 2002.

30. "Pierre Delsuc vu par lui-même ", La Fusée, n 5, Paris, Épi, 1975, p. 146.

31. Citons pour mémoire le célèbre Pour entrer dans le jeu, Paris, SDF, 1935.

32. Tranvouez, Yvon, "Du scoutisme en général et de la Bretagne en particulier ", Scoutisme en Bretagne, Scoutisme breton?, op. cit., p. 20-21. 


\section{Les intrigues}

Elles se ressemblent assez sensiblement en de nombreux points. Des Scouts de France ou des Guides de France ${ }^{33}$, étrangers (parisiens majoritairement) au pays dans tous les cas sauf deux exceptions ${ }^{34}$, mènent une enquête policière aux ingrédients très classiques mais dépaysante. Il s'agit de combattre des contrebandiers de fausse monnaie (L'Étrange aventure de Carnoët), de cigarettes américaines (Toul Ann Héry), de timbres (Lumière sur Kerlivit), ou/et de déjouer les combinaisons d'espions soviétiques ( $L a$ Rude nuit de Kervizel) ou allemands et autrichiens (L'Étrange aventure de Carnoët, Les Fantômes de la chapelle Pol, L'Évadé de Coëtcarantec) ou encore de retrouver les identités perdues de personnages centraux de l'histoire (Une fille pas comme les autres, Gaël des Glénans, Une ténébreuse affaire). Dans deux romans, la Bretagne n'est présente qu'à l'arrière-plan et n'apporte rien de particulier à l'intrigue : il s'agit de La Maison du bord des sables de Jean-Claude Alain et du Signe dans la pierre de Paul Henrys. Dans tous les autres, la province dans toutes ses composantes (population, géographie, folklore, histoire) est essentielle à l'ambiance du récit.

\section{Personnages de Bretagne}

La population bretonne, des années trente aux années soixante, n'a pas une image très positive dans les histoires retenues. Voici par exemple un groupe social populaire, les goémoniers d'Une ténébreuse affaire, qui soupçonnent un trio d'étrangers, les Hennebique installés sur l'îlot voisin, d'assassinat : "L'instinct populaire n'hésita pas un seul instant à prononcer le nom du coupable. Et le dimanche suivant lorsque les deux femmes Hennebique sortirent de l'église les goémoniers leurs firent pendant un kilomètre une escorte silencieuse et pleine de menace $[\ldots]^{35}$. " Plus tard, une épidémie de méningite décime les enfants des goémoniers. Pour eux pas de doute, les coupables sont les Hennebique. La haine grandit et trouve enfin son défouloir lorsque, répondant à l'appel d'une cheftaine croyant son frère et des amis en danger sur l'îlot, le peuple des goémoniers se lance à l'assaut avec une fureur digne des plus féroces jacqueries :

33. Les romans scouts, en général, mettent très peu souvent en scène des éclaireurs et des éclaireuses des autres associations et encore moins des louveteaux, jeannettes, routiers ou guides-aînées. Ces collections s'adressent aux adolescents catholiques en priorité, les guides héritant de la portion congrue. Serge Dalens remarque très judicieusement : "Mais Foncine et Dachs vous le rediraient tout de suite, les filles ne veulent pas de livres écrits pour les filles! Chaque fois qu'on a essayé de faire une collection pour elles, elles l'ont boudée! En revanche, elles veulent des bouquins pour garçons! Au "Signe de Piste", nous avions trois lectrices pour un lecteur. Mais elles aimaient bien qu'il y ait dans le bouquin une fille qui soit plutôt sympathique. " "Commentaire de Serge Dalens ", Ianus Bifrons, op. cit., p. 220-221.

34. Dans L'Etrange aventure de Carnoët, c'est la patrouille des hermines de la $1^{\text {re }}$ Quimper Saint-Patrice qui mène l'aventure. Dans La Maison du bord des sables, une "troupe SaintYves ", d'une localité bretonne indéterminée, est au cœur de l'action. Notons que les héros parisiens des Disparus de Kerlasdouès sont les Cormorans de la troupe Duguesclin. 35. Une Ténébreuse affaire, p. 225. 
"De ferme en ferme, l'amazone lança des paroles enflammées, tout le pays des goémonniers la suivit comme une inspirée. Son succès fut immense. Ils emmenèrent leurs charrettes, les femmes et les enfants suivaient. Ye, l'innocent glapit des cantiques. Une croisade contre Hennebique était déchaînée. Comme un flot grossissant, la cohue prit le chemin des dunes. Une femme força la porte de l'église et sonna le tocsin.

Le glas qui résonna sur la mer crispa les nerfs et acheva de fanatiser ces gens. À leur tête, sur son cheval, la cheftaine galopait. Les hommes à l'avant pour montrer le chemin au suivants mettaient en passant le feu aux tas de goémon. L'air âcre chargé de fumée et d'iode suffoquait. La lande prit feu à son tour. Les chevaux que ces lueurs affolaient, hennirent en courant follement. Ye fit brûler un pot de souffre. Les yeux s'injectaient, la folie monta à toutes les têtes.

- À mort! À mort!

Les cris se firent rauques. Une femme tomba en crise de nerfs. La confusion était à son comble lorsque la horde déferla sur la grève. De l'autre côté, le feu était au fort, le feu était à la tour : l'îlot n'était plus qu'une torche qui rendait le ciel rougeoyant. Sur un des créneaux de la tour, une silhouette s'agitait fantastiquement. Tout ce feu exaspéra la foule qui fonça dans le chenal comme un taureau furieux. [...].

[Les goémoniers prennent pied sur l'îlot.]

- Sus à Hennebique!

La foule fit volte face et se précipita du côté du fort. Comme une vague de raz de marée, la fureur montait. Tapis dans un recoin, trois vieillards muets de terreur attendaient.

[Un homme, allié des pauvres Hennebique, fait alors diversion.]

L'effet fut immédiat. La foule eut d'abord un instinctif mouvement de recul, puis la haine l'emporta. Les hommes eurent des cris aussi sauvages que celui du loup en plein midi. Ils se précipitèrent pour exterminer le démon ${ }^{36}$."

Cet assaut aveugle de tout un peuple, cette " haine stupide du pays ${ }^{37}$ " contre des personnes différentes n'est pas sans rappeler la nouvelle Celui qui avait toujours froid de Claude Seignolle ${ }^{38}$ racontant le meurtre d'un vagabond par dix hommes qui le prenaient pour un revenant parce qu'il dormait dans le cimetière et sentait la mort. Dans les autres histoires, il n'y a pas de descriptions aussi précises d'un groupe de la population bretonne mais l'hérédité est lourde lorsque l'on descend de familles de naufrageurs et que l'on trafique encore pour garder la main! Les populations littorales des Fantômes de la chapelle Pol (Léon) ou d'Une Fille pas comme les autres (Trégor) sont les enfants de ces criminels qui attiraient les bateaux en détresse sur leurs traitres récifs pour dépouiller et massacrer les infortunés marins et voyageurs. Mais il faut bien de l'argent pour faire vivre les familles en charge de "marmailles " ou de " ribambelle de moutards ${ }^{39}$." Quant à l'autorité, on ne l'aime pas et à lire les auteurs on imagine facile-

36. Ibidem, p. 347-348.

37. Ibid., p. 367.

38. SEIgnolle, Claude (dir.), Contes populaires et légendes de Bretagne, Paris, France Loisirs, 1977, p. 211-220.

39. Les Fantômes de la chapelle Pol, p. 26 et p. 33. 
ment la province comme une zone de non droit. La maréchaussée et les gabelous sont totalement déconsidérés et impuissants : "Ils sont si bêtes les gendarmes $[\ldots]^{40}$ " affirme tranquillement une cheftaine à sa servante "plus ou moins fille ou petite-fille de braconniers et de contrebandiers". "La gendarmerie [...] dans ce pays-ci... que voulez-vous qu'elle fasse ${ }^{41}$ ?" répond un bûcheron de Carnoët à un scout de Quimper qui cherche de l'aide pour son enquête. Et le Léon n'a rien à envier à la Cornouaille quand le commandant de Kergro'hen dit exactement la même chose à ces Lyonnais bien naïfs : "Ne faites pas les enfants! Les gendarmes n'arrivent à rien dans un pays comme celui-ci ${ }^{42}$. " Heureusement donc que les scouts sont là pour dénouer les cordages emmêlés de ces éphémérides celtiques.

Peuple violent, primitif, indiscipliné et irrespectueux des lois, les Bretons sont aussi hermétiques avec leur sabir incompréhensible que les Français civilisés écoutent avec amusement, étonnement ou méfiance. Cela donne un charme et un mystère inégalable à la contrée malgré les difficultés de communication : Â un aumônier lyonnais qui avoue de ce peuple aimer " leur simplicité antique qui les fait tutoyer tout le monde, leur parler plein d'un charme savoureux, malgré leurs incroyables inversions de langage et ce méli-mélo de masculin et de féminin qui [le] déroute toujours... ", le propriétaire du lieu de camp répond : "Que voulez-vous mon Père... Ils ne parlent que breton entre eux, et ce qu'ils vous servent en français n'est qu'une traduction mot à mot de la phrase bretonne ${ }^{43}$. " Dans une autre histoire, un scout parisien affirme à son chef de patrouille qui veut interroger la population pour les besoins de l'enquête : "Mais dans les fermes des environs tu ne trouveras que des Bretons incompréhensibles. En outre ils ne voudront pas parler ${ }^{44}$. "Une autre scène pourtant nous présente un petit Breton qui apprend des rudiments de sa langue à un scout de la capitale : "Kenavo... comme ça se prononce. Je ne sais pas comment ça s'écrit mais ça se prononce toujours... Là comme ça... Maintenant, dis comme moi : "Dour mar plich"; ça veut dire : "De l'eau, s'il vous plait ${ }^{45}$." " Ce constat des auteurs de romans, qui souvent, en fait, parlent en connaissance de cause, ayant conduit leur troupe en Bretagne, est corroboré par une cheftaine de louveteaux de la $1^{\text {re }}$ Brest avant guerre, "française " et fille d'officier :

«Dans l'ensemble du Finistère, le breton était la langue normale, unique dans les campagnes : lorsque nous allions en sortie ou au camp, nous n'avions comme interlocuteurs en français, que des enfants allant à l'école; les adultes avaient oublié le peu qu'ils avaient su (quelquefois des jeunes rentrant du service se tiraient d'affaire en français). Pour les femmes, la coiffe du pays - sinon le costume - était toujours porté, et tous les enfants de

40. Une ténébreuse affaire, p. 250.

41. L'Étrange aventure de Carnoët, p. 30.

42. Les Fantômes de la chapelle Pol, p. 93.

43. Ibidem, p. 21.

44. La Rude nuit de Kervizel, p. 59-60.

45. Les Disparus de Kerlasdouès, p. 151. 
Brest, je vous assure, savaient reconnaître une femme de l'île de Sein, une Bigoudène, ou une femme de Quimper [...]. À la messe, dans les villages et les bourgs, nous subissions les sermons et annonces en breton ${ }^{46}$ !"

Les Bretons? un peuple de sous-développés presque aussi exotique que les tribus nègres d'Afrique ou les Indiens d'Amérique pour les scouts français, en fait. Il est exact cependant que le pays reste majoritairement rural et agricole avant le développement rapide des années cinquante et soixante. D'autres pages des romans réservent quand même quelques belles surprises et viennent nuancer ce propos. Voici la galerie des portraits bretons servis dans ces histoires : le hobereau breton tout d'abord, homme ou femme, est le plus typique des personnages. Descendant de chevalier, de corsaire ou de chouans, rebelle et traditionnel à la fois, son nom antique est le témoin du passé de cette " plèbe nobiliaire " indissociable de la terre bretonne. Cultivé mais proche de ses gens qui l'entourent familièrement, il connaît les richesses et les limites de ce peuple. Parfois accompagné de membres de sa famille, il est le propriétaire du lieu de camp et prend plus ou moins d'importance selon les romans. Dans La Rude nuit de Kervizel, Madame de Lanquer est absente de son manoir depuis plusieurs années mais son souvenir est vivace dans la population tandis que dans L'Étrange aventure de Carnoët le baron de Kerhuel " homme de lettre bien connu pour ses études historiques sur la Cornouaille ${ }^{47}$ " a un petit rôle de figuration. Dans Le Signe dans la pierre, roman raider, Lois de Foncroy et Jean-François de Montgranit sont les dignes descendants de croisés bretons du roi saint Louis. Dans quelques autres histoires ce type de personnage est au premier plan. Les Kerlor dans La Maison du bord des sables sont au cœur de l'intrigue ainsi que les Kerlavoz ${ }^{48}$ dans le roman guide Une fille pas comme les autres. Toutefois, le plus beau spécimen reste le commandant comte de Kergro'hen des Fantômes de la chapelle Pol :

"Tout dans l'aspect extérieur de ce dernier, la coupe élégante de la veste, le visage énergique complètement rasé, les cheveux grisonnants taillés en brosse, le regard autoritaire de ses profonds yeux noirs, révélait l'ancien militaire. Comme tous les gens de mer, accoutumés par les éléments à mesurer leurs mouvements et leurs mots, il avait le geste sobre et une parole brève où perçait, malgré son aménité courtoise, un léger soupçon de commandement : officier de marine en retraite, M. de Kergro'hen avait bourlingué sur toutes les mers du globe ${ }^{49}$."

Après tout, le portrait n'est pas si faux que cela et est même assez proche de cette génération d'officier qui donna les commissaires des Scouts de France d'avant guerre. Que l'on songe, si l'on se cantonne à la Bretagne, aux généraux de Salins (Chef Scout), Tabouis (commissaire de Bretagne), de Penfentenyo (commissaire de Cornouaille), aux colonels de

46. Lettre de Françoise Deuve, 2 juillet 1996.

47. L'Étrange aventure de Carnoët, p. 190.

48. "Kerlavoz... la magie des noms! " Une Fille pas comme les autres, p. 18.

49. Les Fantômes de la chapelle Pol, p. 17. 
Réals (commissaire du Léon) et Gridel (commissaire de Saint-Brieuc et Tréguier) et aux autres officiers de la période. Et Kergro'hen, s'il n'est pas scout, prend le scoutisme au sérieux. Aux éclaireurs de la $35^{\mathrm{e}}$ Lyon qui campent en ses terres, il sert de guide détaillant le paysage, expliquant le déchaînement des éléments, initiant les garçons aux joies de la navigation, sur son bateau le Breiz-Izel, et de la pêche aux ormeaux, et enfin luttant avec eux contre les espions et trafiquants de tout poil. La châtelaine des Disparus de Kerlasdouès n'est pas moins originale que Kergro'hen : « La marquise de Botguer chez qui nous campons est une vieille dame... un peu particulière... enfin... depuis la mort de son mari, elle s'est cloîtré dans la propriété et n'en sort que rarement. Elle a peut-être pris au contact de paysans un langage un peu rude et elle fume, paraît-il comme une locomotive $^{50}$ " annonce le chef de troupe à ses scouts. Fortes femmes aussi sont ces châtelaines des autres histoires : Mesdames de Kerlor, maman meurtrie de La Maison du bord des sables; Kerlavoz, veuve éprouvée d'un officier de marine assassiné d'Une fille pas comme les autres et Bourrhis, veuve aussi, vouant " une profonde tendresse [...] exclusive ${ }^{51}$ " à un gamin orphelin, Gaël des Glénans. L'influence de ces maîtresses femmes est originale et reflète sans doute l'importance du matriarcat breton selon les auteurs.

Après cet incontournable de la pastorale de Bretagne, vient le vieux pêcheur ou le vieux paysan selon que l'histoire se place en " armor " (au bord de la mer) ou en " argoat " (dans les terres). Bon ou mauvais, c'est selon, il représente le peuple, une race rustique et sensible, rebelle et fidèle. Le portrait que trace Raymond Baux du Père Urven des Fantômes de la chapelle Pol est un morceau d'anthologie :

"Soudain le père Tellier ${ }^{52}$ bondit en arrière : un petit monstre surgissait des sables sous leurs pieds...

C'était un tout petit vieux, sec comme un fagot d'ajonc. Au fond d'orbites osseuses, sous une broussaille de sourcils sans couleur précise, un œil luisait comme de la braise; sur le second, les paupières boursouflées demeuraient toujours closes. Les piques de sa barbe donnaient à sa face creusée, comme au couteau, de profondes rides longitudinales, l'aspect d'une carapace d'araignée de mer. Ses lèvres rentrantes sur des gencives sans dents maintenaient une courte pipe qu'il venait d'allumer, à l'abri du vent, dans un creux de la dune.

Il était vêtu de l'ample paletot de laine blanche presque brute, à peine plus long que l'étroite et courte veste si caractéristique des paysans bretons, cette sorte de gilet à manches qui avait fait la joie des scouts lyonnais, car ne joignant pas au pantalon à pont, elle laissait échapper tout autour un bourrelet de chemise bariolée du plus plaisant effet. La tête était recouverte du calabousen, passe montagne de drap bleu-marine dont une bande plus claire masquait les quatre coutures qui, des épaules, montaient au sommet du crâne où elles se réunissaient en étoile. Les avant-bras étaient croisés l'un sur l'autre, haut sur la poitrine, et les mains venaient s'engouffrer dans l'ouverture du paletok. D'un vieux pantalon de toile huilée, roulée sous l'ais-

50. Les Disparus de Kerlasdouès, p. 117.

51. Gaël des Glénans, p. 14.

52. L'aumônier des scouts lyonnais. 
selle, sortaient le dard rubicond, les gros yeux noirs furibonds et les longues antennes d'une langouste.

Telle quelle, l'apparition semblait un korrigan d'un autre âge et ne présentait rien de rassurant. Elle justifiait amplement le mouvement de recul du père Tellier ${ }^{53}$."

L'on apprend plus tard que ce Père Urven est une crapule, traître et braconnier qui n'hésiterait pas à donner à manger à ses congres un petit scout trop indiscret. Ne valent pas beaucoup mieux le Père Ferrec de Lumières sur Kerlivit qui séquestre un héros de la résistance et enlève un enfant, ou l'oncle Cosquer de Toul Ann Héry, trafiquant malchanceux et menteur. Dans Gaël des Glénans en revanche, le même auteur, Pierre Delsuc nous présente des marins et des paysans plus sympathiques comme ce Bannalec le fermier au " large sourire " et au " bon rire ${ }^{54}$ ", Guillaume Grannec, le langoustier tourmenté ou encore Jaouen le retraité des pêches du Grand Nord. Ces trois personnages sont les anges gardiens de Gaël, l'enfant à la recherche de son identité.

Maintenant s'avance la femme âgée. Simple ombre fugitive dans Le Signe dans la pierre, elle n'est que la " très vieille bretonne " qui assiste à la messe d'un " prêtre à cheveux blancs ${ }^{55}$ " d'une chapelle isolée. Vivante image de " Da feiz hon tadou $\mathrm{coz}^{56}$ ". C'est dans Toul Ann Héry que Pierre Delsuc peint la vieille la plus typique de nos histoires : "Sous le manteau de la cheminée, une vieille assise sur une chaise basse : c'était la Mamm Goz ${ }^{57}$." Gardienne des traditions familiales et des légendes du pays, elle n'a pas son pareil pour créer une ambiance. D'autres femmes croisent aussi la route des scouts telle cette furie qu'est la Hennebique d'Ouessant (Une ténébreuse affaire) et des commerçantes de village, dépositaires de pain, de cidre et surtout de nouvelles propres à faire avancer les enquêtes : Elles sont cabaretière (Les Disparus de Kerlasdouès), fermière (Les Fantômes de la chapelle Pol), épicière (Lumières sur Kerlivit), ou boulangère (La Rude nuit de Kervizel), et jouent un rôle secondaire mais non négligeable.

Dans trois romans enfin, les auteurs incluent une jeune fille ou un jeune garçon de l'âge des protagonistes, utile auxiliaire à la résolution d'affaires bien compliquées. Du pays, ils guident les étrangers dans leur domaine Dans le roman guide Une fille pas comme les autres, ce personnage est de la famille des propriétaires et scout à Lannion en vacance chez ses parents. Il est aussi de la "grande fraternité scoute " et joue le rôle d'un cousin un peu plus âgé pour les guides parisiennes. Dans les romans scouts, les filles ont une tout autre envergure : jeunes et charmantes, elles ne laissent pas certains indifférents et ajoutent à l'histoire une véritable touche romanesque comme le personnage d'Annick Cosquer dans Toul Ann Héry:

53. Les Fantômes de la chapelle Pol, p. 23-24.

54. Gaël des Glénans, p. 16.

55. Le Signe dans la pierre, p. 81.

56 . « La foi de nos vieux pères ", cantique traditionnel et populaire.

57. La Grand-Mère. Toul Ann Héry, p. 53. 
"Une jeune fille dans l'angle le plus éloigné du pré regardait les scouts en tricotant. Vêtue d'un sarrau bleu, les pieds nus dans des galoches, les cheveux serrés dans un foulard, elle pouvait avoir 16 ans. [...] Elle n'était pas farouche. À la question de Philippe, elle répondit sans hésiter, le fixant de ses yeux couleur de ciel. [...] Son pas était décidé. Pas l'ombre de timidité dans son naturel à marcher près du garçon, et à répondre à ses questions. [...] Sa voix était nette, ses inflexions chantantes selon l'accent cornouaillais. Ses traits fins étaient un peu brouillés par l'abondance des taches de rousseur. Une fille vigoureuse et saine ${ }^{58}$."

Dix ans plus tard, au début des années soixante, Colette Bourrhy, la nièce du maire de Kerlivit, est plus moderne et n'a plus rien à voir avec une petite paysanne : "Elle portait seize ans à peine. Ses cheveux châtains, qu'une permanente tentait vainement de discipliner, étaient en désordre et tombaient sur un front large. Ses yeux d'un bleu pâle et son large rire illuminaient sa figure. Sa robe légère, avec des carreaux jaunes et blancs, lui arrivait un peu au-dessous des genoux. "Elle est bien" songea Henri ${ }^{59}$..." Bien évidemment, dans un roman scout il ne s'agit pas de faire la cour à une jeune fille, si honnête soit-elle, comme dans n'importe quelle historiette. Cela dit, un sentiment fort apparaît parfois selon la volonté de l'auteur. Retrouvons Annick Cosquer après quelques rencontres avec Philippe, le second de patrouille :

" Il était pourtant vrai qu'Annick trouvait Philippe à son goût. C'était bien surprenant alors que tant de choses les séparaient. Mais c'était ainsi. Ce grand et mince garçon était tout à fait son type. Il n'était pas débraillé ainsi qu'affectent de l'être tant de campeurs de cet âge. Son uniforme était net. Il le portait avec simplicité. Il n'avait pas l'air avantageux que prennent neuf sur dix des garçons de la ville. Il s'adressait à elle avec gentillesse Son visage long sur lequel flottait sans cesse une sorte de sourire, son front que barrait une mèche un peu ondulée, son regard chaud surtout, qui semblait vous pénétrer tout entier, autant de traits qui composaient dans l'imagination de la jeune fille un idéal enfin découvert auquel elle s'attachait avec une tranquillité d'esprit d'autant plus grande qu'elle savait bien que cette rencontre d'un jour serait sans lendemain. Elle était fort sensible à certaines qualités de finesse et de distinction. Ceci ne saurait surprendre que ceux qui ignorent la Cornouaille et la vieille race celte qui l'habite. Ce n'est pas en vain que l'air y est tout de limpidité et de lumière, que les nuages qui passent font traîner des ombres fugitives et changeantes. Cela façonne une sensibilité de qualité ${ }^{60}$."

Amour courtois entre les deux adolescents condamnés à la séparation de la fin du camp : Philippe, nouveau chevalier, donnera à sa douce, devant le front de sa patrouille, un foulard de son unité que la jeune Cornouaillaise nouera en fichu avant de s'évanouir dans la campagne, le cœur aussi gros que celui de son soupirant.

58. Toul Ann Héry, p. 45 sq

59. Lumière sur Kerlivit, édition de 1988, p. 117.

60. Toul Ann Héry, p. 103-104. 
Le dernier point à noter est l'absence quasi totale du clergé local. Les églises et les chapelles ne sont plus desservies ou si rarement et les recteurs ne sont que des souvenirs d'ancien régime. Le si chrétien peuple breton semble abandonné à ses croyances et ses superstitions et les prêtres n'a aucune part dans ces romans si ce n'est par les aumôniers français qui accompagnent les unités en Bretagne. Bretagne, terre des prêtres? certainement pas dans la littérature scoute.

\section{Les lieux}

Presque toutes les histoires étudiées se déroulent en Basse Bretagne ainsi que le montre le tableau ci-dessous :

\begin{tabular}{|l|l|l|}
\hline Roman & Environs de... & Département \\
\hline La Rude nuit de Kervizel & Douarnenez, Cap Sizun & Finistère sud \\
Une Ténébreuse affaire & Brest & Finistère nord \\
Les Disparus de Kerlasdouès & Landerneau & Finistère nord \\
L'Étrange aventure de Carnoët & Quimperlé, Bénodet & Finistère sud \\
Les Fantômes de la chapelle Pol & Kerlouan & Finistère nord \\
Traon-Ar-Rhun & Morlaix & Finistère nord \\
L'Évadé de Coëtcarantec & Manque de précision & Morbihan \\
La Maison du bord des sables & Étel? & Morbihan \\
Une fille pas comme les autres & Trégastel & Côtes-du-Nord \\
Le Signe dans la pierre & Baud & Morbihan \\
Patrouille ardente & Saint-Malo & Ille-et-Vilaine \\
Toul Ann Héry & Vallée du Goyen & Finistère sud \\
Gaël des Glénans & Pont-L'Abbé, Archipel des Glénans & Finistère sud \\
Lumière sur Kerlivit & Douarnenez & Finistère sud \\
\hline
\end{tabular}

Pour les auteurs, la Bretagne authentique est à l'ouest, toujours plus à l'ouest, en Cornouaille et Léon. À eux deux, ces pays sont le cadre de neuf aventures sur quatorze, le Morbihan l'est de trois et les Côtes-du-Nord d'une seule. L'est du pays, la Haute Bretagne de langue gallo, est ignorée, à l'exception de la "république " de Saint-Malo. il n'est certainement pas suffisamment dépaysant. Rétrécissons le cercle : la plupart des histoires se passent en armor, sur le littoral, voire sur l'eau et dans des îles comme c'est le cas dans Les Fantômes de la chapelle Pol (l'île Kerlouan), Les Disparus de Kerlasdouès (l'île de Botguer), Une Ténébreuse affaire (une île au large de Brest), et Une Fille pas comme les autres (l'île de Costaérès au large de Trégastel). Lîle bretonne est un des lieux mythiques par excellence dans l'imaginaire scout, où la Bretagne ne peut-être qu'océane. La mer, évidemment omniprésente, est indissociable du paysage ce qui est particulièrement sensible dans Les Fantômes de la chapelle Pol, Une fille pas comme les autres et davantage encore dans Gaël des Glénans, roman maritime s'il en est ${ }^{61}$. Curieusement cependant, aucun roman scout marin ne se passe en Bretagne alors que les troupes y sont nombreuses. Jérôme, le scout

61. Collection « Signe de Piste », Paris, Alsatia, 1959. 
d'Une ténébreuse affaire est bien scout marin mais cette qualité n'est spécifiée qu'une fois dans le roman, nonobstant le fait qu'il sache fort bien godiller et nager. Seul Pierre-André Bernard dans Le Bachi ${ }^{62}$, mettra en scène la Bretagne, mais seulement dans les souvenirs de ses héros, Jacques et Guy de Kervern, fils d'officier de marine, anciens chefs marins à Brest, exilés en Normandie.

Le plus souvent, les héros s'installent sur le littoral et découvrent la civilisation de cet étonnant milieu pour des citadins de grandes villes. La mer et le rivage peuvent alors se présenter sous différents aspects au lecteur : hostile comme au début des Fantômes de la chapelle Pol ou accueillant pour les balades des guides d'Une fille pas comme les autres. Aux auteurs de créer l'ambiance. Raymond Baux excelle dans le style : "Les fanions claquaient au souffle du noroit qui balayait, sans répit, la côte du Finistère depuis cinq jours. Le sable fin, arraché des grèves, courait à ras le sol et cinglait les mollets nus. Chassés des récifs submergés, leur habituel refuge, pétrels et goëlands affamés ramaient dans la bourrasque et s'épandaient sur les champs labourés en nappes blanches et criardes ${ }^{63}$."

Pierre Delsuc s'entend aussi à décrire ces tempêtes qu'il connaît bien mieux que quiconque mais il sait également peindre la sérénité de la baie de Douarnenez et le pittoresque de l'archipel des Glénans. C'est ce même auteur qui nous livre le roman d'argoat le plus complet, le plus réaliste et aussi le plus mystérieux :

«Depuis le matin la Patrouille était seule dans un pays inconnu d'elle. Sa route suivait une ligne de crêtes qui découvrait au nord toute la région de Douarnenez, hormis la ville elle-même trop éloignée et trop basse pour qu'on l'aperçût. Au sud, c'était la vallée du Goyen, couverte de landes désertiques jusqu'aux lointaines régions de Plozevet et de Pouldreuzic. Cette vallée constituait en fait la zone d'exploration du Cygne. Peu creusée, à pentes douces et arrondies piquetées çà et là de boqueteaux de pins, recouvertes partout d'un épais tapis de genêts, de bruyères et d'ajoncs, elle offrait à perte de vue un monotone paysage ondulé. Mais la lumière qui la baignait, légère et claire, lui conférait une sorte d'irradiation envoûtante. Une brise marine courait sur la lande, qu'elle agitait de larges reflets mobiles sous un soleil un peu voilé. On paraissait se mouvoir dans un monde irréel ${ }^{64}$."

La mission de la patrouille est la suivante : "Relever toute trace de monument, quel qu'il soit, témoignant de l'antiquité de la civilisation de ce pays : tel que dolmen, menhir, vieux calvaire, petites chapelle, fontaine ${ }^{65}$." L'observation n'est pas de tout repos car, les vieilles pierres et les monuments remarquables abondent, qui jouent un rôle important dans l'histoire. Il en va de même pour plusieurs des autres romans. Presque tous ont leur antique manoir, chapelles à souterrains et crypte, fermes, moulins, ports, croix de chemin. Dans quelques cas, ces monuments sont au centre de l'in-

62. Collection "Signe de Piste ", Paris, Alsatia, 1959.

63. Les Fantômes de la chapelle Pol, p. 5.

64. Toul Ann Héry, pp. 25-26.

65. Ibidem. 
trigue et donnent leur sens et le titre au roman. Ainsi les chapelles Pol et de Toul Ann Herry, l'église de Kerlivit, le manoir de Kervizel, l'abbaye SaintMaurice de Carnoët. Les auteurs sont de bons observateurs; ils sont des chefs scouts formés à la topographie et à l'orientation. Les descriptions sont crédibles même si parfois, pour la cohérence du récit, ils prennent quelques libertés avec la réalité, inventant un lieu ou en transposant un autre. Pierre Delsuc, en particulier, arrange des lieux et en complète d'autres dans ces pays bigouden et glazic qu'il connaît parfaitement : le cimetière du bourg de Plogastel-Saint-Germain n'est plus depuis bien longtemps autour de son église ${ }^{66}$ et l'institution Saint-François-Xavier est plutôt le grand pensionnat de Vannes que celui de Quimper ${ }^{67}$. De même, la chapelle Notre-Dame de la Clarté de Toul Ann Héry n'est-elle pas sur la vallée du Goyen mais plus au sud, sur la paroisse de Pluguffan. Michel Renouard invente quant à lui un Kerlivit en pays douarneniste mais l'authentique est sur la commune d'Argol. La campagne bretonne, diverse et riche, se prête de bonne grâce à ses fantaisies d'auteurs.

En revanche, la ville n'est pas pour les scouts, qui sont faits pour la nature et les grands espaces. Lointaine, elle n'est que peu évoquée. On y arrive pour aller au camp; on en repart pour rentrer chez soi. La ville n'est qu'une gare, un point de passage. Elle garde aussi le souvenir de l'école. Elle n'a pas d'intérêt donc pour des histoires de vacances. De plus, elle peut présenter un danger. C'est en ville, à Douarnenez, qu'un gamin se fait enlever, dans l'indifférence quasi générale (Lumière sur Kerlivit). Ce n'est pas étonnant, après tout, lorsque l'on connaît la réputation de ce port de pêche cité dans quatre histoires : c'est dans La Rude nuit de Kervizel, alors que des scouts tentent de sauver un ingénieur russe pisté par la Tcheka, que le sort de Douarnenez est définitivement réglé pour la postérité scoute : « Il n'y a rien d'impossible, répondit le scoutmestre, à ce que les Soviets entretiennent partout des agents secrets. Précisément Douarnenez est une des citadelles du communisme en France. La municipalité de cette ville ne demanderait pas mieux, j'imagine, que de prouver à ses maîtres de Moscou l'excellente qualité de son rouge $[\ldots]^{68}$. " Le port sardinier est en effet la première municipalité de France à s'être dotée d'une municipalité communiste et pour longtemps. Lorsque le chef scout rédige son roman en 1927, la ville est devenue, depuis le dur conflit de 1924-1925 entre ouvriers et usiniers, un symbole de la résistance au patronat pour le communisme et le syndicalisme en Bretagne et France. Elle l'est aussi pour l'autre camp et représente un danger certain. Il reste donc notable que ce qui est consi-

66. Gaël des Glénans, p. 22.

67. Ibidem, p. 171.

68. La Rude nuit de Kervizel, p. 114. Dans la dernière édition (Saint-Vincent-sur-Oust, ELOR, coll. "Les Jeux de l'aventure ", 1996, p. 97.), on ne cite plus uniquement Douarnenez mais on évoque de manière plus floue " plusieurs villes bretonnes ". Sur l'image de Douarnenez, voir la thèse de géographie de Jean-Michel Le Boulanger, Douarnenez de 1800 à nos jours, essai de géographie historique sur l'identité d'une ville, Institut universitaire européen de la mer, 1999. 
déré comme le premier roman scout se soit ainsi radicalement marqué et ancré dans la réalité politique de son temps, mêlant le grand jeu scout de ces nouveaux chevaliers au péril rouge oriental et athée. Dans le thème de l'anticommunisme primaire, Delsuc s'il est un des premiers, est bientôt imité par plusieurs auteurs catholiques. Le très célèbre Tintin au pays des Soviets, paru trois ans après La Rude nuit de Kervizel, en 1930, est emblématique. L'étonnant Les Trois contre Moscou (1931) du chanoine brestois René Cardaliaguet qui conte la lutte de jeunes catholiques en Bretagne occupée par les communistes chinois paraît également chez Spes dans la collection "Des fleurs et des fruits " dirigée par l'abbé Félix Klein, comme l'ouvrage de Pierre Delsuc.

\section{L'ambiance}

Les personnages et les lieux nous ont déjà fait rentrer dans une ambiance particulière. Des thèmes transversaux donnent aux romans scouts un goût de terroir inimitable qui tient aussi sans doute au cidre, au pain beurre et aux crêpes dont nos héros sont largement abreuvés et nourris tout au long des pages. Dans plusieurs cas, l'intrigue est liée à un moment de l'histoire de Bretagne. Deux périodes sont ici privilégiées : la persécution antireligieuse sous la Révolution et la résistance durant la deuxième guerre mondiale. En revanche, pas un mot, ou si peu, sur le Moyen Âge, le temps de l'indépendance ou la Bretagne province. Ces épisodes sélectifs correspondent toujours aux clichés, réels ou supposés, sur la Bretagne vue de France. C'est encore Pierre Delsuc qui nous donne les pages les plus fortes sur le sujet, autant dans Toul Ann Héry pour le premier thème que dans Gaël des Glénans pour le second. Comme les garçons de la patrouille du Cygne groupés dans la salle commune autour de l'âtre, écoutons la Mamm Coz :

"Sous la Grande Terreur, quand nos Messieurs Prêtres se cachaient pour ne pas mourir, quand les églises toutes fermées elles étaient, et les cloches muettes donc, les truands étaient les maîtres et exterminaient tout ce qui parlait du Bon Dieu. Pourtant les bonnes gens en pèlerinage ils allaient, à Notre-Dame de la Clarté, jusqu'à l'église, oui donc. Ils n'entraient point dedans, mon père m'a répété souventes fois, mais dessous. Là Monsieur L'Hostis, d'Audierne, venait leur dire la Messe. Comme si les aveugles pouvaient attendre, ma Doué, et tous les bonnes gens qui souffrent. Alors ils venaient quand même, et même quand les Bleus ont brûlé tout le pays et l'église elle-même. Quand l'église brûlait encore en terre la Messe était dite pour les malheureux pécheurs. Notre-Dame de la Clarté n'a jamais été abandonnée, même qu'il en coûtât vie. Gardons-lui révérence, mes fils, et que Dieu nous préserve de ces temps de malheur ${ }^{69}$."

Dans Une Fille pas comme les autres revient aussi le thème du clerc persécuté par les républicains. Par contre, on ne parle, ni dans l'un ni dans l'autre, de chouannerie, de guérilla. La contrée apparaît non comme une

69. Toul Ann Héry, p. 60. 
terre de révolte, comme la Vendée militaire par exemple ${ }^{70}$, mais comme une victime subissant les événements.

La Deuxième Guerre mondiale est aussi une époque de mémoire dans les romans. Dans ceux de l'immédiat après-guerre, traumatisme oblige, le souvenir est vivace et de nombreux " méchants ", trafiquants, crapules ont un lien avec l'outre Rhin. Pierre Fuval après avoir, dans Le Mystère du lac de Laffrey ${ }^{71}$ (1938), réglé leurs comptes aux anarchistes haineux et ennemis des scouts, combat les espions nazis du Morbihan à Berlin dans L'Évadé de Coëtcarantec. Dans Les Fantômes de la chapelle Pol, le trafiquant se nomme Muller et est un ancien officier allemand; dans L'Étrange aventure de Carnoët enfin, le chef de bande est un autrichien. Simplisme de l'époque. Dans quelques autres, des familles sont endeuillées par des pertes imputables aux bombardements meurtriers de notre région (Les enfants Kermor, la marraine de Gaël). Il faut bien attendre une dizaine d'années pour qu'une certaine sérénité se dégage.

Après l'Histoire, les légendes. Les auteurs guides d'Une fille pas comme les autres donnent le ton : Pour elles, la Bretagne est " un pays étrange, secret et nostalgique entre tous ${ }^{72}$ ". Ces deux cheftaines, Jacqueline Royer et Simone La Selve, tout en ayant un certain don de l'intrigue et de l'écriture, sont des professionnelles de la commande régionaliste. Leurs romans, qui paraissent dans Guide de France, sont des soutiens de la politique des commissaires nationales des années cinquante. Une Fille pas comme les autres (ou An Ano Kolet), est le roman breton. L'Inconnue de Valcluse a illustré auparavant la Provence et Le Dragon blanc est l'opus alpin. Les auteurs écrivent un guide touristique à la main et servent aux lectrices tous les poncifs attendus (pardon, calvaire, ossuaire, danses) avec un bonheur certain. Quelques légendes sont rapportées mais sans beaucoup de détails : celle d'un fantôme au manoir et celle de Notre-Dame de la Clarté près Perros. Au contraire, Raymond Baux et Pierre Delsuc s'en donnent à cœur joie dans leurs livres pour faire frissonner les scouts au rythme de la légende de la mort. Chez l'un, c'est l'histoire du prêtre errant cherchant un servant pour sa messe des morts ${ }^{73}$ et plus loin le récit d'un intersigne ${ }^{74}$, l'enterrement nocturne d'un infortuné; chez l'autre c'est celle, plus classique, des lavandières de la nuit ${ }^{75}$. Le dépaysement est complet tant pour les héros que pour le lecteur.

70. Voir les romans pour jeunes, mais non scouts, de Henri Bourgenay, Sang et Or, Paris, Alsatia, coll. "Signe de Piste », 1954 et de X.-B. Leprince, Guillery de Saint-Gril, Paris, Alsatia, coll. « Rubans Noirs », 1966.

71. Fuval, Pierre, Le mystère du lac de Laffrey, Paris, Alsatia, coll. « Signe de Piste », 1938.

72. Une Fille pas comme les autres, p. 14.

73. Voir Fouquet, Dr A., " La cathédrale ", dans Seignolle, Claude (dir.), op. cit., p. 316320. Le conte de Charles Corre collecté par Anatole Le Braz, "La messe des âmes ", Scout, $\mathrm{n}^{\circ}$ 45, 20 novembre 1935, p. 495-496, est assez proche de cette légende.

74. « Les intersignes annoncent la mort. [...] Les intersignes sont comme l'ombre, projetée en avant de ce qui doit arriver " écrit Anatole Le Braz, op. cit, p. 103

75. Voir SouvESTRE, Émile, "Les lavandières de la nuit », dans SEIGNOLLE, Claude (dir.), op. cit., p. 288-297. Cette légende est aussi signalée dans Le Signe dans la pierre, p. 90. 
Au terme de ces lectures, la Bretagne transparaît bien comme une province à l'identité fortement marquée. Bien sûr d'autres parties de l'hexagone qui sont étudiées dans les articles ou qui sont traitées dans les collections, et dont nous avons pu avoir connaissance, affirment elles aussi leurs particularités et leur particularisme. Cependant, elles sont rares celles qui, comme en Bretagne, développent une si complète mythologie. Dans l'imaginaire des chefs et des garçons, soutenu par cette littérature scoute, ce pays fut dès les origines, et reste encore, une destination privilégiée des camps scouts et guides. Contrée romanesque et romantique depuis le travail des érudits et des écrivains du XIX ${ }^{\mathrm{e}}$ siècle, terre primitive chargée d'histoire, de foi et de fidélité, ou supposée telle, pays à la géographie tourmentée et variée, la Bretagne ainsi simplifiée ne pouvait que convenir aux nouveaux chevaliers du scoutisme catholique en quête de Graal, comme aux raiders prêts à affronter tous les déchaînements des tempêtes de terre et de mer. Les thèmes des grands jeux s'en trouvaient invariablement définis entre Gaule celtique et Table Ronde, guerre de Succession et Chouannerie, contrebande et résistance. Le mythe dépassant la réalité, la Bretagne était alors élevée au rang de composante essentielle des camps d'été et de la littérature scoute.

\section{RÉSUMÉ}

Le scoutisme, mouvement de jeunes fondé au siècle dernier, est une méthode d'éducation active par la nature qui utilise largement l'imaginaire de l'adolescent. Une importante production écrite (articles de la presse scoute, romans) a servi de support à cet imaginaire. Cette étude analyse la place et la perception très particulières de la Bretagne et des Bretons au travers du genre original de la littérature scoute. Sur quarante années, en suivant quelques héros dans leurs pérégrinations, une Bretagne se révèle, contrée fascinante et mystérieuse, cadre idéal de grands jeux et d'aventures de papier.

\section{ABSTRACT}

Scouting is a youth organization in the last century. It is an educational method based on nature and using children's imagination. A great number of writter documents such as articles and novels has helped develop this world of fantasy. Our study analyses the part played by Brittany and Bretons in this original kind of literature and the way it was perceived. Our analyses of a few fictitions heroes and their peregrinations over a period of forty years shows that Brittany is a fascinating and mysterious land and therefore an ideal setting for a games and adventures. 